\title{
A METHOD FOR THE DIRECT OBSERVATION OF RETINAL VESSELS IN THE EXPERIMENTAL ANIMAL*
}

\author{
BY \\ CHRISTOPHER PEDLER \\ Department of Pathology, Institute of Ophthalmology, University of London
}

IN the original studies of Ashton and his co-workers on the effect of high ambient oxygen concentrations on the blood vessels of the immature retina (Ashton, Ward, and Serpell, 1953, 1954), a method was devised whereby it was possible to observe the retinal vessels of an anaesthetized animal through a stereoscopic microscope (Ashton and Cook, 1954). This technique, primarily designed for work in connexion with the aetiology of retrolental fibroplasia, clearly has many other applications both within and beyond the field of ophthalmological research, since it provides a fairly straightforward means of directly observing and correlating retinal vascular changes with other physiological data.

The apparatus used for this work has now been modified and developed; many of the earlier technical difficulties have been overcome and the new methods have proved entirely satisfactory in numerous experiments carried out during the past year and in the preparation of a film. We have, therefore, decided to publish a detailed account of the apparatus and technique at present in use in this laboratory.

\section{Apparatus}

Fig. 1 (opposite) shows that the main frame is constructed from steel scaffold tubing, which properly stressed provides an immensely rigid framework and easily supports all the main units. These comprise the lighting system, animal chamber, microscope, ciné camera, and control gear.

Lighting System.-The source used (B, Fig. 2, overleaf) is a 4-mm. Tungsten arc of very high intensity surrounded by a steel box, which, although primarily acting as a screen for stray ultra-violet irradiation, also affords some protection in case of a tube explosion, for, although we have never experienced this, it is a distinct possibility, and the arc should never be mounted in an unshielded position. The arc housing is fixed soaxially to a telescope tube, which contains a heat-absorbing glass, paired condensers, and a final biconvex lens. The convergent beam of light which emerges from this is brought to a point of focus approximately $1 \mathrm{~cm}$. behind the reflecting surface of the plane mirror (B, Fig. 3, overleaf). It is then reflected downwards through a right angle past the glass of the eye chamber (C, Fig. 3) and onto the retina (D, Fig. 3). The diameter of the spot of light thus produced in the eye can be varied by the iris diaphragm built into the lens tube.

* Received for publication December 3, 1956. 


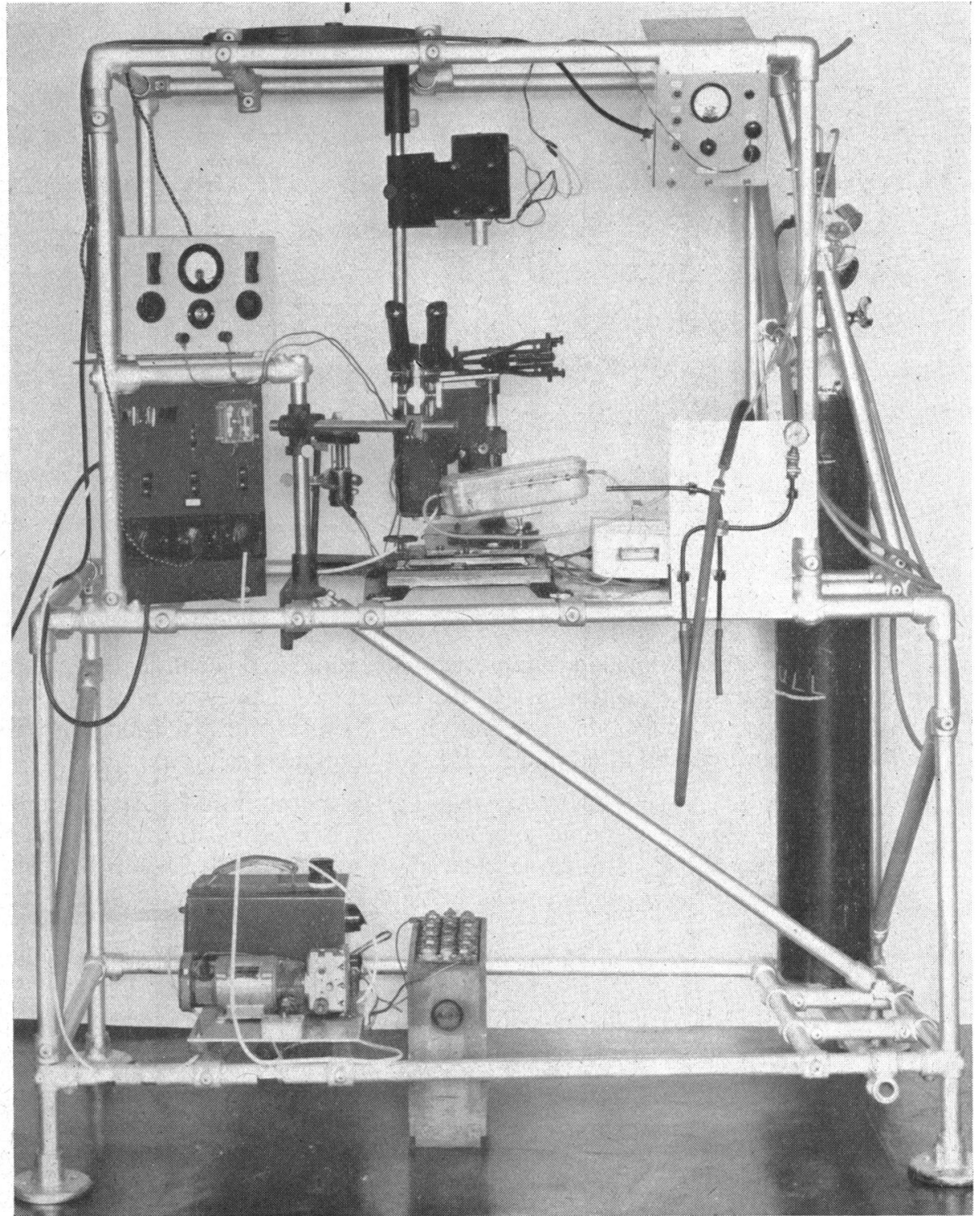

FIG. 1.-General view of apparatus.

Animal Chamber (Fig. 4, overleaf).- This is made from a commercially obtainable plastic food container, and is essentially a rectangular box with an airtight lid divided transversely, so that the section over the animal's head can be removed separately. This part also has a small hole cut in its centre to coincide with the position of the rim of the eye chamber (vide infra). On opposite sides of the box there are two tube connexions, one connected to the oxygen supply via a flowmeter and reduction valve, and the other leading to a B.O.C. oxygen analyser. This device 


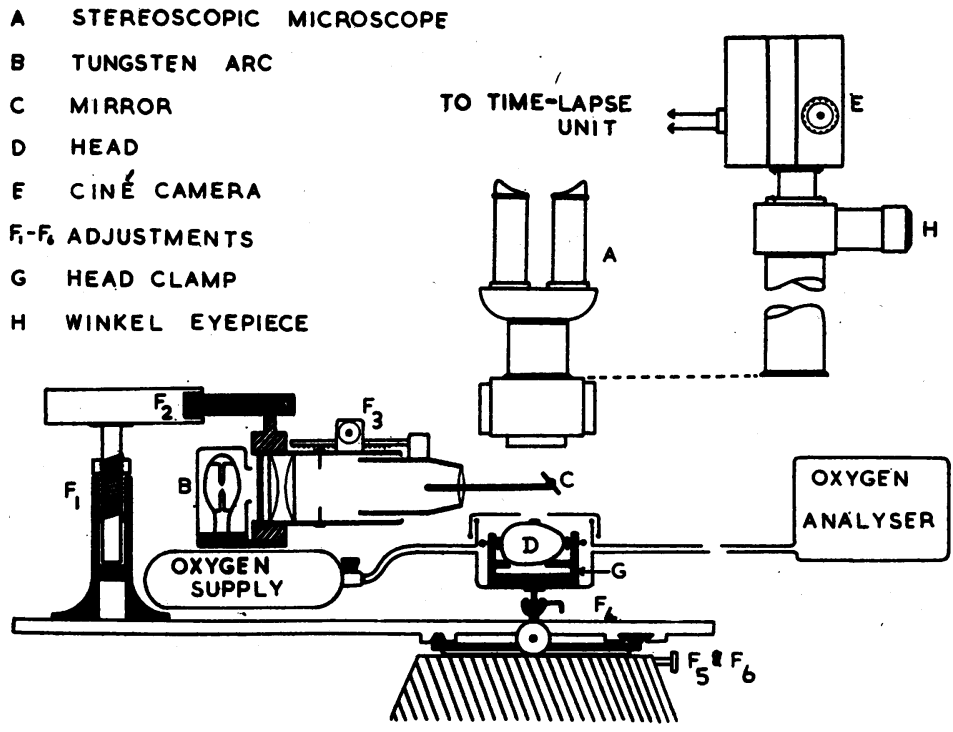

Fig. 2.-Diagram of essential components.

which operates on a diamagnetic principle, provides rapid and accurate readings of the ambient oxygen concentration inside the chamber. The floor of the box is attached to the base plate carrying the lamp unit, by means of a self-locking universal joint so that it can be tilted and fixed in any oblique position.

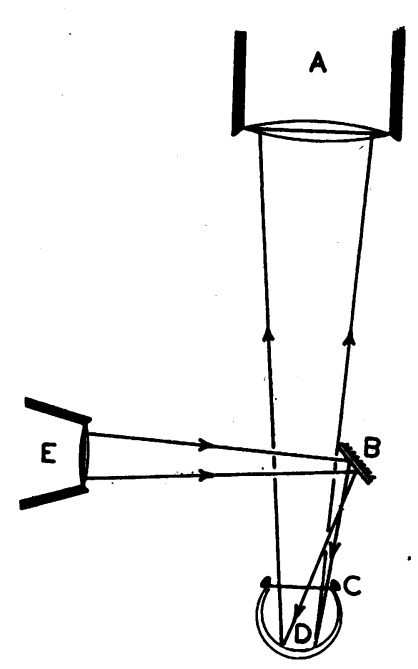

Frg. 3.-To show method of illumination.

Microscope.-This is a conventional Zeiss stereoscopic microscope (A, Fig. 2 and 3) mounted on a horizontally-swinging arm suspended from the main frame, so that its action together with the normal up and down movement of the focusing adjustment provides the necessary universal movement. Normally, a binocular head is used, but this is easily detachable, and can be quickly replaced by the Winkel eyepiece and ciné camera (E and H, Fig. 2).

Ciné Camera.-The first camera to be used was a Ciné Kodak special, fixed to the main frame so that it operated over one eyepiece of the binocular head. This was fitted with an electrically-operated shutter release mechanism, which in turn was connected to a simple electromechanical time lapse unit. Using this linkage, a shutter speed of about one frame every 2-6 sec. was used over periods of up to half an hour.

A second camera unit has now been built, consisting of a modified G.S.A.W. Gun Camera fixed to a Winkel eyepiece which fits into the base of the stereoscopic microscope.

Controls.-The adjustments necessary for the correct positioning of the components have already been mentioned, and are in fact largely self-explanatory on 


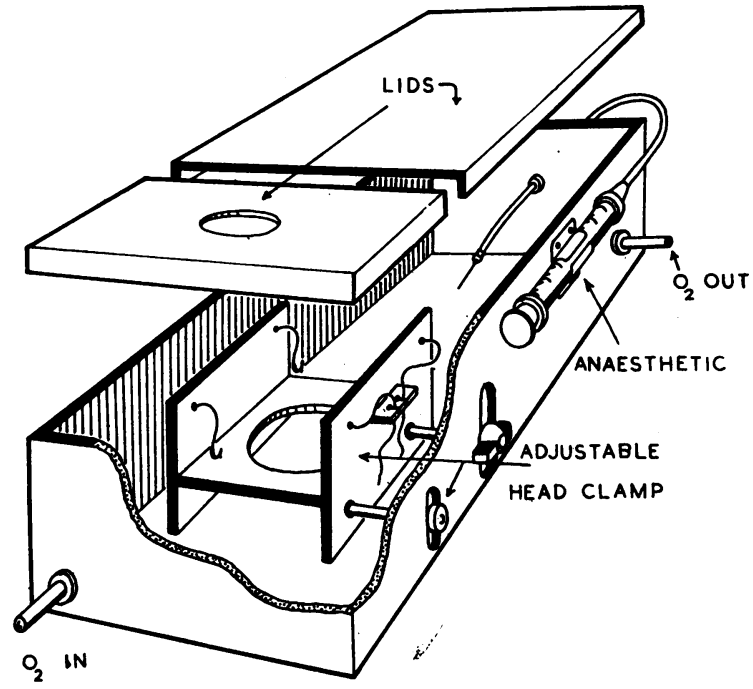

Fig. 4.-Animal chamber.

reference to Fig. 2. The only part requiring a separate note is the time lapse unit for the camera. Essentially, this consists of a motor-driven gearbox (F, Fig. 5) with an intermittent final drive rotating the disc (G, Fig. 5) through one quarter of a revolution per second. On the disc are mounted four projecting rods, three of which are of different length, so that when the disc rotates, the contacts $\mathrm{J}$, which lead to the camera solenoid, are alternately made and broken. Thus if a different camera speed is required, the screws (L) are loosened and the contacts moved away from, or nearer to the disc, causing a different number of projecting pegs to strike the shutter contacts as the disc rotates.

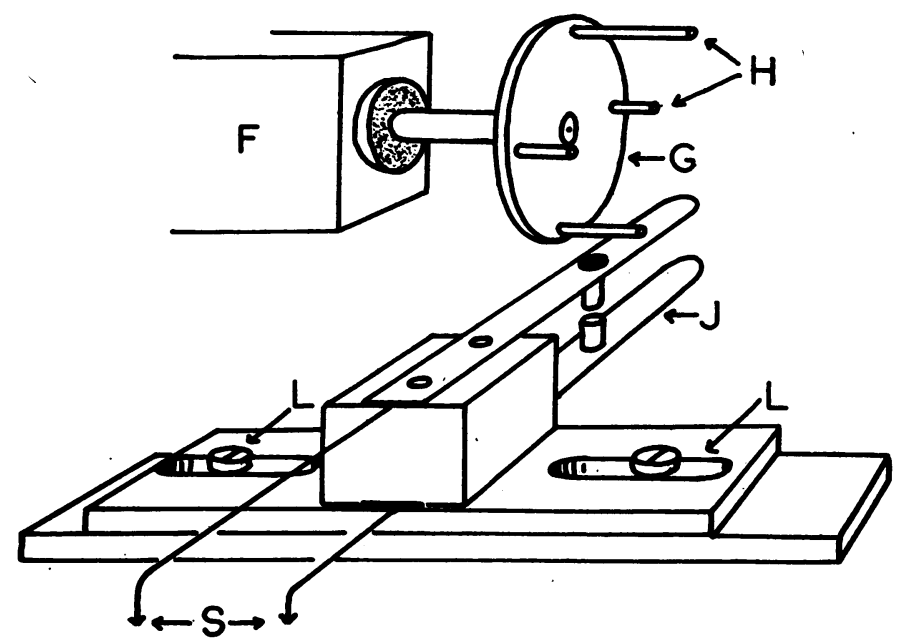

Fig. 5.-Time lapse unit. 
Technique

Before the animal can be fixed in the oxygen chamber, it is necessary to remove the anterior ocular structures and replace them by a cylindrical metal chamber containing a flat glass disc. The method of performing this operation has not changed since it was first devised and the only modification which has been made is in the design of the "limbal window", details of which can be seen in Fig. 6.

A

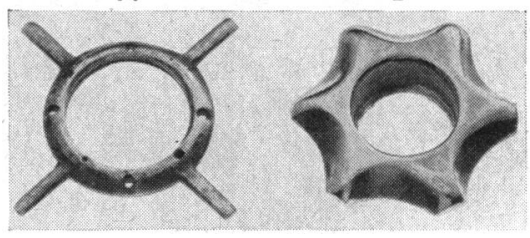

Fig. 6.-Limbal window.

After removal of the eyelids and conjunctiva, the brass ring (A, Fig. 6) is secured to the limbus by four sutures inserted through holes in its rim. The cornea is then removed with a cutting diathermy knife and the lens extracted, leaving behind the posterior part of the capsule as a temporary means of retaining the vitreous. Finally the inner chamber (B, Fig. 6) is screwed into the outer ring and the glass on its under surface allowed to impinge on the anterior vitreous face. In this way, the pressure exerted on the remaining intra-ocular contents may be varied within fairly wide limits, for if intra-ocular pressure is not maintained, then the retina will detach completely within a short time.

Once this "limbal window" operation is completed, the animal is transferred to the plastic chamber, and its head fixed rigidly by the clamps (G, Fig. 2). A needle is then inserted intraperitoneally and connected via a short length of polythene tube to a Mantoux syringe clipped to the outside of the box. This provides a convenient way of administering further doses of anaesthetic without disturbing the animal or the apparatus. Finally the airtight lid already referred to is applied and relative positions of the mirror, box, and microscope altered until optimum illumination of the retina is obtained. In this way the retinal vessels can be studied for as long as $10 \mathrm{hrs}$ continuously at magnifications varying between 26 and 66 times.

I am most grateful to Dr. Norman Ashton for his help and encouragement during the preparation of this paper, and also to Dr. Peter Hansell for his help with the photographic apparatus described. I should also like to thank Miss E. FitzGerald for clerical assistance and Mr. G. Knight for his valuable technical help.

\section{REFERENCES}

Ashton, N., and Cook, C. (1954). British Journal of Ophthalmology, 38, 433.

, WARD, B., and SERPELL, G. (1953). Ibid., 37, 513. (1954). Ibid., 38, 397. 